\title{
Wavefront coherence time seasonal variability and forecasting at the San Pedro Mártir site
}

\author{
E. Masciadri and T. Garfias \\ Instituto de Astronomía, Universidad Nacional Autónoma de Mexico, Apartado Postal 70-264, 04510 D.F., Mexico \\ Received 30 August 2000 / Accepted 14 November 2000

\begin{abstract}
In order to efficiently use the recent astronomy high angular resolution techniques (Adaptive Optics and Interferometry) to correct the perturbed wavefront arriving at the telescope pupil, it is necessary to characterize a set of astroclimatic parameters. One of these is the wavefront coherence time $\tau_{\mathrm{AO}}$. It is an integral parameter, defining the maximum temporal correlation of the perturbed wavefront and it depends on the optical turbulence $C_{\mathrm{N}}^{2}(x, y, z)$ and the wind intensity $|\boldsymbol{V}(x, y, z)|$ in the whole troposphere. In this paper we use an atmospheric non-hydrostatic model (Meso-NH) conceived to simulate the classical meteorological parameters $(p, T$ and $\boldsymbol{V})$ and adapted to simulate the optical turbulence $\left(C_{\mathrm{N}}^{2}\right)$ to characterize the $\tau_{\mathrm{AO}}$ in a region of some kilometers around the astronomical site of San Pedro Mártir (SPM) in Baja California (Mexico). We study the seasonal variability of the wind intensity in the whole atmosphere $(20 \mathrm{~km})$ above the SPM site during one year. We show that, using the $C_{\mathrm{N}}^{2}$ profiles simulated by Meso-Nh initialized with ECMWF (European Center for Medium Weather Forecasts) data, we obtain typical $\tau_{\mathrm{AO}}$ values in the $\mathrm{V}$ band $(\lambda=0.5 \mu \mathrm{m})$. We calculate the seasonal variability of the $\tau_{\mathrm{AO}}$ in SPM and also some preliminary results about the seasonal variability of $C_{\mathrm{N}}^{2}$. Moreover, we suggest a physical explication of these variabilities. Finally we investigate the possibility of forecasting $\tau_{\mathrm{AO}}$.
\end{abstract}

Key words. atmospheric effets - site testing - instrumentation: interferometers; adaptive optics

\section{Introduction}

A detailed characterization of the atmospheric optical turbulence is necessary in order to successfully apply the modern techniques of High Angular Resolution observations such as Adaptive Optics and Interferometry. Actually, a set of astroclimatic parameters are used to describe the atmospheric turbulence state (the seeing $\varepsilon$, the wavefront coherence time $\tau_{\mathrm{AO}}$, the scintillation rate $\sigma_{\mathrm{I}}^{2}$, the isoplanatic angle $\theta_{\mathrm{AO}}$ and the spatial wavefront outer scale $\mathcal{L}_{0}$ ). These parameters provide different information. For example the seeing, which is the integral of the turbulence ( $C_{\mathrm{N}}^{2}$ profiles) along lines of sight, gives us quantitative information about the total turbulence in the atmosphere. The wavefront coherence time $\tau_{\mathrm{AO}}$ gives the velocity turbulence characteristics. For a single turbulence shear moving with a horizontal velocity $V, \tau_{\mathrm{AO}}$ is the time that the wavefront needs to cover the distance of $r_{0}$. This last parameter is the wavefront spatial coherence that is the typical size over which the wavefront perturbations are correlated. $\tau_{\mathrm{AO}}$ fixes the minimum exposure time for a detection system if we want a free temporal filtering signal. In the case of Interferometry (Buscher 1994; Davis \& Tango 1996; Roddier \& Lena 1984), if the wavefront

Send offprint requests to: E. Masciadri,

e-mail: elena@astroscu.unam.mx perturbations change consistently during the exposure time, the fringe visibility is reduced and this can result in an incorrect image interpretation. In the case of Adaptive Optics, if the correction system frequency is lower than $\left(\tau_{\mathrm{AO}}\right)^{-1}$ we obtain only a partial wavefront correction. In this article we consider the following expression of the Fried parameter $r_{0}$ (Roddier 1981):

$r_{0}=\left[0.423\left(\frac{2 \pi}{\lambda}\right)^{2} \int_{0}^{\infty} C_{\mathrm{N}}^{2}(h) \mathrm{d} h\right]^{-3 / 5}$,

which is proportional to $(\varepsilon)^{-1}$, and of the wavefront coherence time (Roddier et al. 1982):

$\tau_{\mathrm{AO}}=0.31 \frac{r_{0}}{v_{\mathrm{AO}}}$,

where $v_{\mathrm{AO}}$ is the horizontal equivalent velocity:

$v_{\mathrm{AO}}=\left[\frac{\int_{0}^{\infty}|\boldsymbol{V}(h)|^{5 / 3} C_{\mathrm{N}}^{2}(h) \mathrm{d} h}{\int_{0}^{\infty} C_{\mathrm{N}}^{2}(h) \mathrm{d} h}\right]^{3 / 5}$.

We should note that many different definitions of $\tau_{\mathrm{AO}}$ exist in the literature (Greenwood 1977; Tango \& Twiss 1980; 
Table 1. Typical $\tau_{\mathrm{AO}}$ values related to different observation bands and different seeing conditions. In the computation of $\tau_{\mathrm{AO}}$ a typical value of $v_{\mathrm{AO}}=12(\mathrm{~m} / \mathrm{s})$ (see the text) has been used

\begin{tabular}{c|cc|cc|cc|cc}
\hline \hline Wavelength & \multicolumn{3}{|c|}{$V$} & \multicolumn{2}{c|}{$K$} & \multicolumn{2}{c|}{$M$} & \multicolumn{2}{c}{$N$} \\
\hline$\lambda(\mu \mathrm{m})$ & \multicolumn{2}{|c|}{0.5} & \multicolumn{2}{c|}{2.2} & \multicolumn{2}{c}{4.8} & \multicolumn{2}{c}{10} \\
\hline$\varepsilon(\operatorname{arcsec})$ & $r_{0}(\mathrm{~cm})$ & $\tau_{\mathrm{AO}}(\mathrm{msec})$ & $r_{0}$ & $\tau_{\mathrm{AO}}$ & $r_{0}$ & $\tau_{\mathrm{AO}}$ & $r_{0}$ & $\tau_{\mathrm{AO}}$ \\
\hline 2 & 5 & 1.5 & 29.6 & 8.88 & 75.45 & 22.63 & 182.05 & 54.61 \\
1 & 10 & 3 & 59.2 & 17.76 & 150.9 & 45.27 & 364.10 & 108.23 \\
0.7 & 15 & 4.5 & 88.8 & 26.64 & 226.35 & 67.90 & 546.15 & 163.84 \\
0.5 & 20 & 6 & 118.4 & 35.52 & 301.8 & 90.54 & 728.20 & 218.46 \\
\hline \hline
\end{tabular}

Buscher 1988; Nightingale \& Buscher 1991). The wavefront coherence time values can be different depending on the kind of filter function used to compute $\tau_{\mathrm{AO}}$. We refer to the Buscher (Buscher 1994) for a more exhaustive discussion about this.

Equations $(2-3)$ show how $\tau_{\mathrm{AO}}$ depends on the vertical spatial distribution of the turbulence and on the wind intensity. It is easy to see that, for the same detector, a low turbulence concentrated in very fast shears can have a stronger effect on the observations than a larger turbulence distributed in slow shears. Moreover, $\tau_{\mathrm{AO}}$ is a chromatic parameter which depends on the wavelength to a $6 / 5$ power. In Table 1 typical $\tau_{\mathrm{AO}}$ values related to different observational bands and to different seeing conditions are listed. We computed $\tau_{\mathrm{AO}}$ using a typical value of $v_{\mathrm{AO}}$ $=12 \mathrm{~m} / \mathrm{s}$ (Vernin et al. 2000).

The detectors normally used in adaptive optics work with an average exposure time $\tau_{\mathrm{e}} \in[1-20]$ msec. In Table 1, one sees that, for the medium and far infrared range ( $M$ and $N$ bands) $\tau_{\mathrm{AO}}$ is greater than $\tau_{\mathrm{e}}$. In this case therefore, the signal is not filtered temporally. In the near-infrared range ( $K$ band), if we are in a good site $(\varepsilon \leq 0.7 \operatorname{arcsec}), \tau_{\mathrm{AO}}$ is still greater than $\tau_{\mathrm{e}}$. In the optical range ( $V$ band), on the contrary, the typical $\tau_{\mathrm{AO}}$ is in the $[1-10]$ msec range. In this case, $\tau_{\mathrm{AO}}$ can determine the temporal filtration in adaptive optics and interferometry applications.

It is difficult to measure $\tau_{\mathrm{AO}}$ because the signal can easily be filtered during the measurements. If the exposure time is larger than the $\tau_{\mathrm{AO}}$, all the frequencies higher than $1 / \tau_{\mathrm{e}}$ can be eliminated in the signal. Some instruments were recently employed to measure $\tau_{\mathrm{AO}}$, for example the technique based on fringe visibility measurements of the Sydney University Stellar Interferometry (SUSI) (Davis 1996), the Scidar technique (Vernin et al. 2000; Avila et al. 2000) and the Generalized Scale Monitor (GSM) (Martin et al. 2000). However, a systematic monitoring of the $\tau_{\mathrm{AO}}$ has never been done in any astronomical observatory. The advantage of numerical atmospherical models is that they can give the $C_{\mathrm{N}}^{2}(x, y, z)$ and wind intensity $\mid \boldsymbol{V}(x$, $y, z) \mid$ profiles sampled with the vertical resolution of the model levels in a region around the telescope. Moreover, the numerical models can provide, at the same time, a 2D spatial characterization, a seasonal characterization and a forecasting of $\tau_{\mathrm{AO}}$.
In this study we use a non-hydrostatic atmospheric model (Meso-NH) (Lafore et al. 1998) adapted to simulate the optical turbulence (Masciadri et al. 1999a). The ability of the model to simulate $C_{\mathrm{N}}^{2}$ profiles was successfully tested in previous articles (Masciadri et al. 1999b; Masciadri et al. 2000a). The typical spatial and temporal fluctuations of the wind are larger than the optical turbulence fluctuations so it is relatively easy to simulate the wind profiles with a meteorological model such as Meso-Nh.

In Sect. 2 we investigate the wind intensity seasonal variability in the whole atmosphere $(20 \mathrm{~km})$ over the San Pedro Mártir region during one year (1997). To do this, we use ECMWF (European Center for Medium Weather Forecasting) meteorological data (MARS catalog). We prove that the $C_{\mathrm{N}}^{2}$ profiles simulated by the model provide typical values of $\tau_{\mathrm{AO}}$ for $\lambda=0.5 \mu \mathrm{m}$. In Sect. 3 we study the importance of the $C_{\mathrm{N}}^{2}$ in the $\tau_{\mathrm{AO}}$ simulations and, in Sect. 4 , we investigate the possibilities of forecasting $\tau_{\mathrm{AO}}$.

\section{Wind and $\tau_{\mathrm{AO}}$ seasonal variability}

In order to characterize the wind intensity in the whole troposphere $(20 \mathrm{~km})$ in a region above the San Pedro Mártir Observatory (31.0441 N, 115.4569 W) we studied 156 vertical profiles of analysis wind intensity provided by the ECMWF climatological model (horizontal resolution of 0.5 degrees). These profiles are evenly distributed over the 1997 year and are computed at 00:00 hours U.T. on each night in the geographic coordinates $(31 \mathrm{~N}, 116 \mathrm{~W})$. In the following we will call this point $\mathrm{AE}$ (analysis extracted). More information about the analysis can be found in the Annex of (Masciadri et al. 1999b). Although the distance between the AE point and the Observatory is about $50 \mathrm{~km}$, we can assume that, above $4 \mathrm{~km}$ (altitude), the analysis wind is representative of the flow above the Observatory. The AE point is at a $553 \mathrm{~m}$ above the ground, the San Pedro Mártir site is at $2780 \mathrm{~m}$ above the ground. We prefered to choose AE in the west side respect with the astronomical site because it is in an upstream position in respect to the wind. At these altitudes the wind temporal and spatial fluctuations scales are larger in the horizontal direction than in the vertical one so we judge that this hypothesis is acceptable for a seasonal wind characterization. In the first $4 \mathrm{~km}$ above the ground, which are affected by orographic effects and are therefore not well described by the analysis data, we 

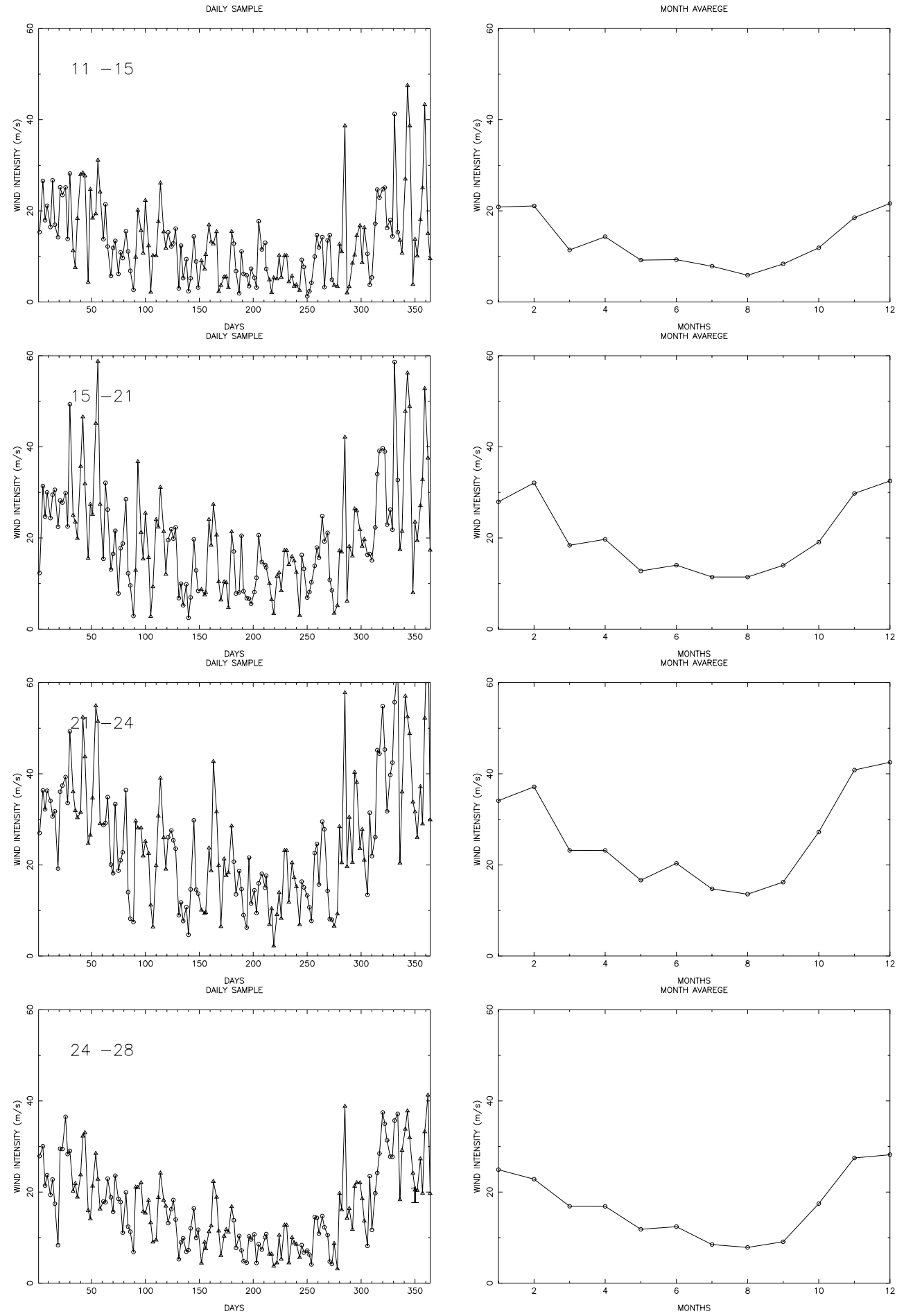

Fig. 1. Daily (left column) and monthly (right column) wind intensity values during the 1997 year in the (31 N, $116 \mathrm{~W})$ grid point. From top to bottom: [4-7] km, [7-11] km, [11-14] km and [14-18] km from the ground. The numbers on the top left corner (left column) are the model levels corresponding to the different altitudes expressed in kilometers

use wind intensities measured at the San Pedro Mártir Observatory (Alvarez 1969).

We averaged the wind intensity in 4 different atmospheric regions [4-7] km, [7-11] km, [11-14] $\mathrm{km}$ and [14$18] \mathrm{km}$ over the whole year. In Fig. 1 are shown the daily values (left column) and the monthly values (right) averaged over each region. A clear seasonal variability is evident in each atmospheric region, especially in the right column where the daily fluctuations are filtered. During the (December - February) months one can observe a stronger wind than during the (July - September) months. In Fig. 2 we show the vertical wind profiles averaged over the winter period (December - February: right) and over the summer period (July - September: left). At an 

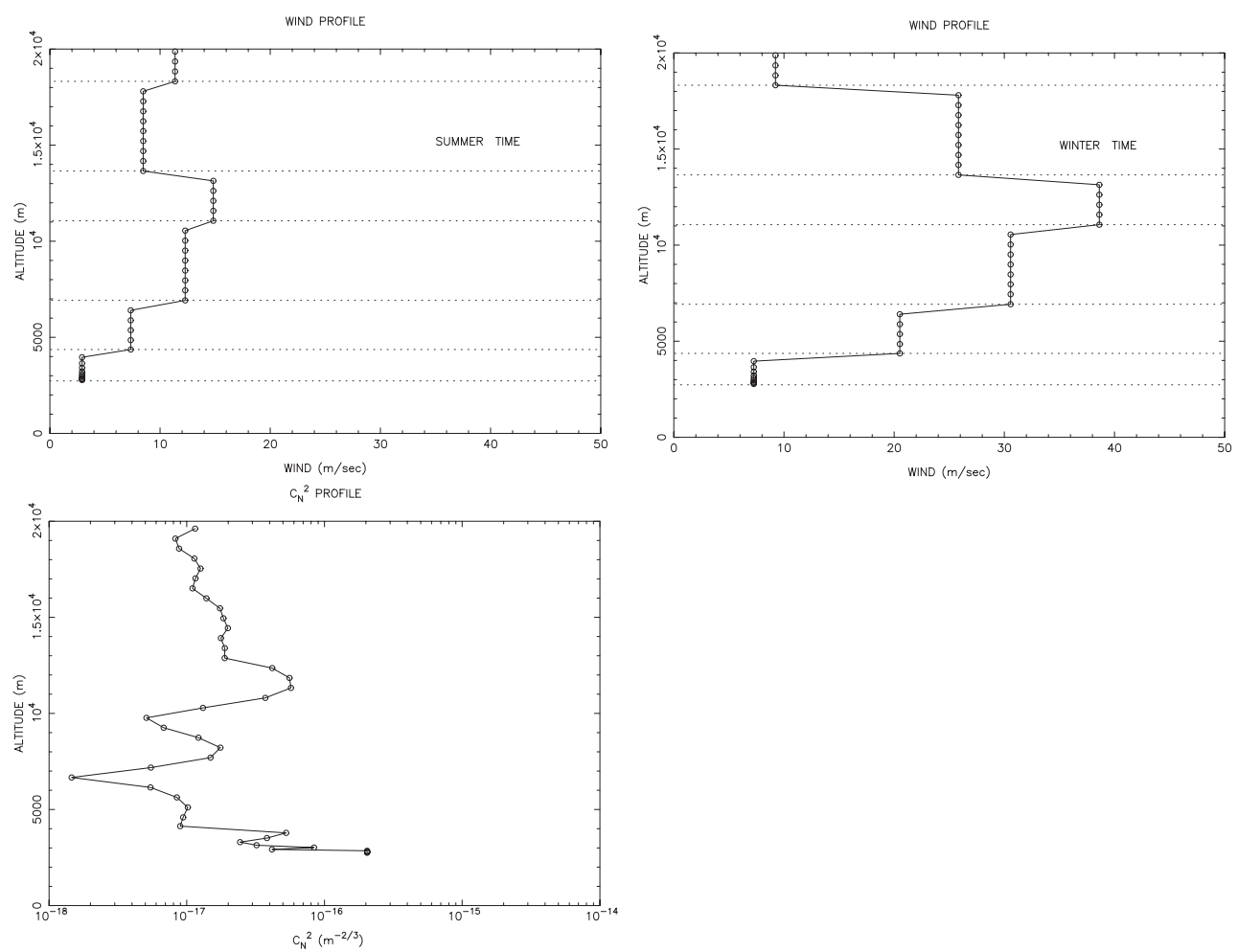

Fig. 2. At the top the vertical wind profiles averaged over the summer time (July - September: left) and over the winter time (December - February: right) are shown. The values are provided by the ECMWF analysis computed in the (31 N, $-116 \mathrm{~W})$ grid point at 00:00 hours in 156 nights sampled over the 1997 year. At the bottom a $C_{\mathrm{N}}^{2}$ profile simulated by the Meso-Nh model over the San Pedro Martir Observatory is shown

altitude of about $12 \mathrm{~km}$, that is the region with the strongest wind, one can observe a typical difference between the two periods of about $25 \mathrm{~m} / \mathrm{s}$. This is in a good agreement with the values found in general climatological studies (Holton 1992). In the first $4 \mathrm{~km}$ we used the values of $7.26 \mathrm{~m} / \mathrm{s}$ and $2.92 \mathrm{~m} / \mathrm{s}$, respectively, for the winter and summer period provided by a climatological wind study done by Alvarez (1969).

In order to compute $\tau_{\mathrm{AO}}$ we use a typical $C_{\mathrm{N}}^{2}$ profile simulated by Meso-Nh (also shown in Fig. 2). This $C_{\mathrm{N}}^{2}$ profile is related to a seeing of $0.65 \operatorname{arcsec}$ and is obtained by averaging the simulation over 3 hours in the same night. We used vertical $(p, T$ and $\boldsymbol{V})$ profiles provided by ECMWF data to initialize Meso-Nh. More details about the Meso-Nh initialization technique can be found in Masciadri et al. (1999a). We find, for the winter period $\tau_{\mathrm{AO}}=1.79 \mathrm{msec}, v_{\mathrm{AO}}=27.18 \mathrm{~m} / \mathrm{s}$, and for the summer period $\tau_{\mathrm{AO}}=5.75 \mathrm{msec}, v_{\mathrm{AO}}=8.30 \mathrm{~m} / \mathrm{s}$. The spatial and temporal fluctuations of the optical turbulence are smaller than the ones of the classical meteorological parameters like the wind velocity and they are not considered in this estimation. In the same way we do not consider in this section the $C_{\mathrm{N}}^{2}$ seasonal variability in the sense of variations induced by climatological large scale phenomena. We note that no studies of the $C_{\mathrm{N}}^{2}$ seasonal variability in the troposphere during such a long period have ever been published. We therefore make the following hypothesis: that the high frequency $C_{\mathrm{N}}^{2}$ fluctuations exist and that they are equally distributed in the year and that the intensity of these fluctuations can modify locally the $\tau_{\mathrm{AO}}$ value but do not modify the seasonal variability of $\tau_{\mathrm{AO}}$. In the following section we will describe this in a more detailed way.

We therefore conclude that, under these conditions, the model can simulate typical values of $\tau_{\mathrm{AO}}$ in the visible range. At the same time, we find that in the San Pedro Mártir site, $\tau_{\mathrm{AO}}$ has a clear seasonal variability. We estimate a $\Delta \tau_{\mathrm{AO}}=3.96 \mathrm{~ms}$ difference between the summer and winter time.

\section{Discussion}

Here we try to answer the following two questions:

- Can the $C_{\mathrm{N}}^{2}$ vertical distribution affect locally the $\tau_{\mathrm{AO}}$ value? Here locally means in a temporal scale smaller than a season, for example a night or, better, a part of a night;

- Can the $C_{\mathrm{N}}^{2}$ vertical distribution affect the season variability of $\tau_{\mathrm{AO}}$ ?

In order to answer to the first question we show an example in Fig. 3. These are the simulation outputs that we obtained over the Paranal site (Chile) in a precedent study (Masciadri et al. 1999b). We choose this example because it is representative of what we want to prove.

In the left column the outputs related to the 16/5/93 night and in the right column the 25/5/93 night ones are 

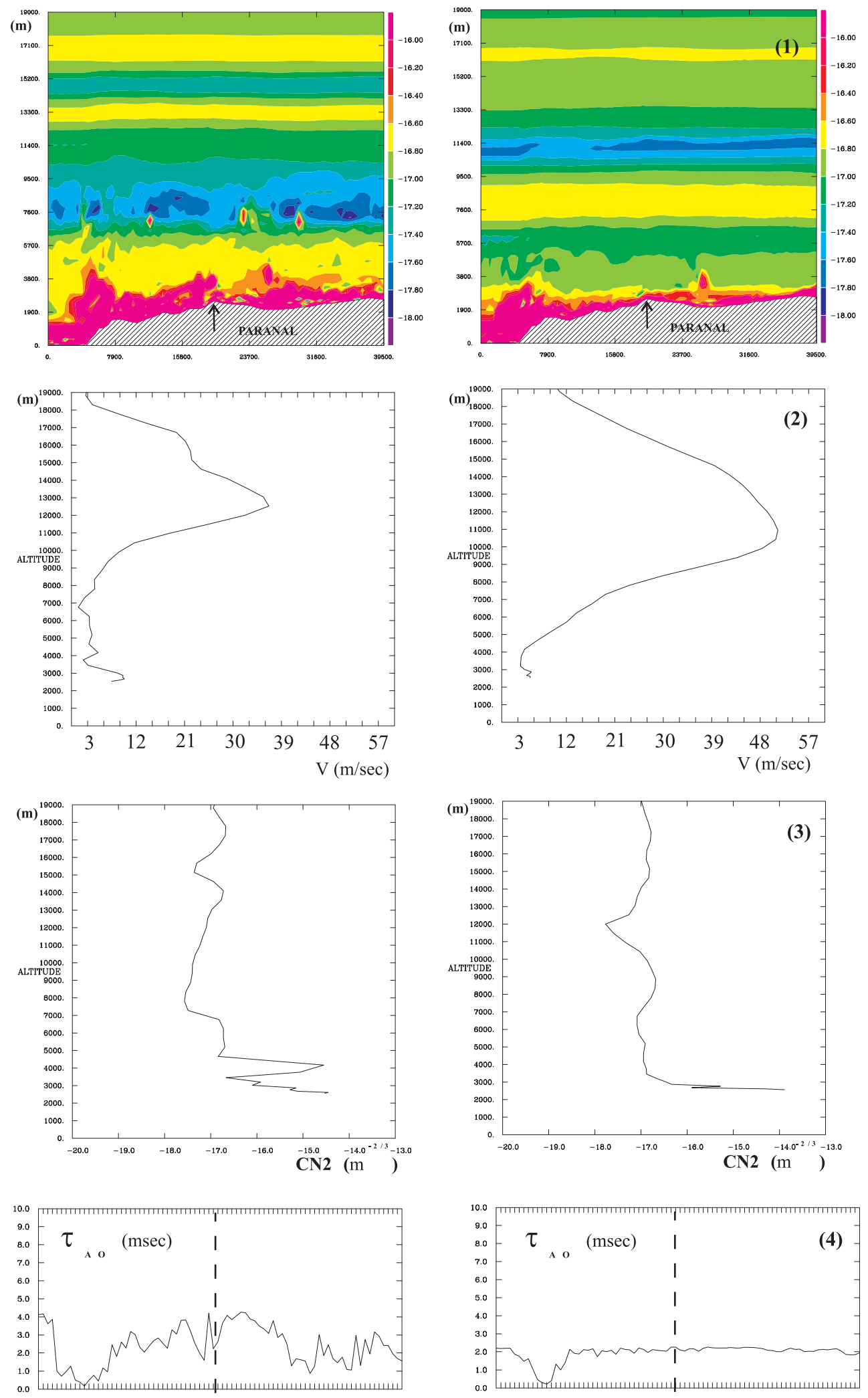

Fig. 3. Simulation outputs related to the $16 / 5 / 93$ night (left column) and 25/5/93 night (right column) done above the Paranal site (Chile). From top to bottom: 1) the vertical sections of $C_{\mathrm{N}}^{2}$ isolines. The color table (in the Web) shows a strong $C_{\mathrm{N}}^{2}$ near the ground and a lower and stratified $C_{\mathrm{N}}^{2}$ above $5 \mathrm{~km} .2$ ) -3 ) the vertical wind and $C_{\mathrm{N}}^{2}$ profiles simulated above the Paranal site. 4) the vertical $\tau_{\mathrm{AO}}$ sections centered above the Paranal site. (This figure is available in color in electronic form) 
Table 2. Integrated parameters $\left(\varepsilon, \tau_{\mathrm{AO}}\right.$ and $\left.v_{\mathrm{AO}}\right)$ computed above the Paranal (Chile) related to the simulations of $16 / 5 / 93$ and 25/5/93 nights shown in Fig. 3

\begin{tabular}{ccccc}
\hline \hline & NIGHT & $\tau_{\mathrm{AO}}(\mathrm{msec})$ & $v_{\mathrm{AO}}(\mathrm{m} / \mathrm{s})$ & $r_{0}(\operatorname{arcsec})$ \\
\hline$[0-20] \mathrm{km}$ & $16 / 5$ & 2.44 & 6.51 & 5.6 \\
& $25 / 5$ & 2.29 & 24.46 & 22 \\
\hline \hline$[0-5] \mathrm{km}$ & $16 / 5$ & 3.08 & 5.29 & 5.7 \\
& $25 / 5$ & 19.26 & 4.89 & 35 \\
\hline \hline$[5-20] \mathrm{km}$ & $16 / 5$ & 4.35 & 17.24 & 85 \\
& $25 / 5$ & 2.32 & 31.06 & 32 \\
\hline \hline
\end{tabular}

shown. From top to bottom we show: the vertical sections (East-West direction extended over $40 \mathrm{~km}$, the Paranal site is in the centre) of the $C_{\mathrm{N}}^{2}$ isolines (1), the vertical wind intensity and $C_{\mathrm{N}}^{2}$ profiles simulated above the Paranal site (2)-(3). Finally, the vertical sections of $\tau_{\mathrm{AO}}$ along the same direction (4) are shown. The general characteristics of these simulations are: horizontal resolution of $500 \mathrm{~m}$ for a surface of $20 \times 60 \mathrm{~km}$.

In Table 2 we give the integral parameters $\left(r_{0}, \tau_{\mathrm{AO}}\right.$ and $\left.v_{\mathrm{AO}}\right)$ computed above the Paranal site from these two simulations. One can see (first two lines of Table 2) that the optical turbulence is stronger in the $16 / 5$ night (the values of $r_{0}$ are very different in the two nights). On the contrary, the values of $\tau_{\mathrm{AO}}$ are comparable in the two nights. In the last column one can observe that the turbulence is distributed in a homogeneous way over the whole troposphere in the $25 / 5$ night. On the contrary, in the $16 / 5$ night the turbulence is concentrated near the ground. The wind intensity difference between the two nights at $12 \mathrm{~km}$ is of about $15 \mathrm{~m} / \mathrm{s}$. The wind intensity near the ground is comparable and not higher than $10 \mathrm{~m} / \mathrm{s}$. How do the wind and the $C_{\mathrm{N}}^{2}$ contribute to the $\tau_{\mathrm{AO}}$ estimation? One can observe that the $\tau_{\mathrm{AO}}$ has comparable values in the [5$20] \mathrm{km}$ region and over the whole atmosphere in the $25 / 5$ night. On the contrary, in the $16 / 5$ night, the $\tau_{\mathrm{AO}}$ in the [5-20] $\mathrm{km}$ region is two time larger than over the whole atmosphere. This means that in the $25 / 5$ night the strong wind at high altitudes that principally affects the $\tau_{\mathrm{AO}}$. In the $16 / 5$ night, on the contrary, the strong turbulence near the ground determine the values of $\tau_{\mathrm{AO}}$. Comparing the $[0-5] \mathrm{km}$ and the $[5-20] \mathrm{km}$ regions in the $16 / 5$ night, one can see that $r_{0}$ increases by a larger factor $(85 / 5.7 \sim 15)$ than $v_{\mathrm{AO}}(17.24 / 5.29 \sim 3)$.

We can therefore answer the first question, concluding that $C_{\mathrm{N}}^{2}$ can affect locally the $\tau_{\mathrm{AO}}$. In the example shown, the strong turbulence near the ground is probably caused by an orographic effect more easily related to a local meteorological condition than to general climatologic phenomena

It is more difficult to answer to the second question. The $C_{\mathrm{N}}^{2}$ is characterized, at the same time, by two different kind of fluctuations: the natural high frequency turbulence fluctuations and a slower seasonal fluctuation. Here we are interested to the second one. Measurements extended over 3 years in the San Pedro Mártir Observatory (Echevarría et al. 1998) show a seasonal variability of the seeing, which seems to be better during the summer time. The seeing is an integrated parameter so we cannot know a priori whether this variability is caused by large scale phenomena or by local ones. The large scale phenomena principally affect the $C_{\mathrm{N}}^{2}$ at high altitudes while the short scale phenomena affect the $C_{\mathrm{N}}^{2}$ at low altitudes. We have computed, using the Meso-Nh model, a set of simulations (4 nights in the summer time and 4 in the winter time). We computed (Table 3) the seeing contributions at high altitudes $\left(\varepsilon_{\mathrm{FA}}\right)$, at low altitudes $\left(\varepsilon_{\mathrm{BL}}\right)$ and over the whole $20 \mathrm{~km}$ range $\left(\varepsilon_{\mathrm{TOT}}\right)$. In the summer time, the average seeing is $\left\langle\varepsilon_{\mathrm{FA}}\right\rangle=0.38$ arcsec. In the winter time we obtain $\left\langle\varepsilon_{\mathrm{FA}}\right\rangle=0.55$ arcsec. Moreover, each night shows the same tendency at high altitudes: a larger optical turbulence during the winter time. This preliminary result shows a seasonal $C_{\mathrm{N}}^{2}$ variability at high altitudes at large climatological scales. In the last column of the Table 3 are reported the $\tau_{\mathrm{AO}}$ obtained using the wind profils of Fig. 2 . In the winter, the average $\tau_{\mathrm{AO}}$ is equal to $1.41 \mathrm{msec}$, in the summer is equal to $6.89 \mathrm{msec}$. The difference of $\tau_{\mathrm{AO}}$ between winter and summer time previous estimated (3.96 msec) is therefore enhanced $(5.48 \mathrm{msec})$. This results indicate that the seasonal variability of the $C_{\mathrm{N}}^{2}$ probably affect the $\tau_{\text {AO }}$ seasonal variability. Obviously, this same experiment should be extended to the whole year to have a richer statistical sample, but it is not possible at the present time because of limited computational resources.

To give an idea of the $\tau_{\mathrm{AO}}$ modulation over the astronomical site we show in Fig. 5, as an example, a simulated $\tau_{\text {AO }}$ map related to the 27 July 1997 night. The whole surface is $60 \times 60 \mathrm{~km}$ centered on the Observatory. The black lines are the level isolines (orographic structure). On the right of the Observatory, along a North-West versus South-East direction, one can observe the maximum slope mountain chain. The gray scale shows different $\tau_{\mathrm{AO}}$ values. We observe small $\tau_{\mathrm{AO}}$ values above the maximum slope, that is the place where the turbulence production is probably larger. All around the Observatory, the $\tau_{\mathrm{AO}}$ map presents modulations in a range of about 6 msec.

We suggest now a physical explication of the $C_{\mathrm{N}}^{2}$ seasonal variation. We know that the deterministic turbulence production in the atmosphere depends on the Richardson number:

$R_{\mathrm{i}}=\frac{g}{\theta} \frac{\partial \theta / \partial z}{(\partial U / \partial z)^{2}}$ 
Table 3. Seeing simulated by the Meso-Nh model during 4 nights in the summer time and 4 nights in the winter time during the 1997 year above the San Pedro Mártir Observatory

\begin{tabular}{ccccc}
\hline \hline NIGHT & $\varepsilon_{\text {TOT }}(\operatorname{arcsec}):[0-20] \mathrm{km}$ & $\varepsilon_{\mathrm{BL}}:[0-1] \mathrm{km}$ & $\varepsilon_{\mathrm{FA}}:[1-20] \mathrm{km}$ & $\tau_{\mathrm{AO}}(\mathrm{msec})$ \\
\hline $26 / 07 / 97$ & 0.58 & 0.39 & 0.37 & 6.57 \\
$27 / 07 / 97$ & 0.53 & 0.31 & 0.38 & 7.17 \\
$28 / 07 / 97$ & 0.57 & 0.33 & 0.41 & 6.68 \\
$30 / 07 / 97$ & 0.53 & 0.34 & 0.36 & 7.17 \\
\hline $25 / 12 / 97$ & 1.28 & 1.09 & 0.53 & 0.9 \\
$26 / 12 / 97$ & 0.77 & 0.38 & 0.61 & 1.51 \\
$27 / 12 / 97$ & 0.72 & 0.42 & 0.53 & 1.51 \\
$28 / 12 / 97$ & 0.67 & 0.34 & 0.53 & 1.73 \\
\hline \hline
\end{tabular}

where $\theta$ is the potential temperature and $U$ is the horizontal wind component. $R_{\mathrm{i}}$ describes the stability level of the atmosphere. In the night time, the atmosphere is generally stable $\left(R_{\mathrm{i}}>1 / 4\right)$ and it is difficult to detect the dynamical instability $(\partial U / \partial z \gg 1$ and $\partial \theta / \partial z<$ $1)$ that causes the turbulence in strongly stratified shears. Following a statistical approach (Van Zandt et al. 1978; Van Zandt et al. 1981) we can replace the deterministic $R_{\mathrm{i}}$ with a probability density function describing the stability and instability factors in the atmosphere provided by climatological data. In this stochastic context it was proved (Van Zandt et al. 1978) that the probability function to find dynamical instabilities depends on the wind and potential temperature vertical gradient at large scales (of a few kilometers). In Fig. 2 it is evident that during the winter time the vertical wind gradient is stronger than during the summer time. In Fig. 4 we show the potential temperature vertical profiles obtained averaging the ECMWF data described in Sect. 2 over the winter period (December February, bold line) and over the summer period (July September, thin line). The potential temperature gradient is not too different during the winter and summer time. We therefore conclude that, during the winter time, the probability to generate dynamical instabilities is higher than during the summer time. We find a further confirmation of this hypothesis in the Cerro Pachón site campaign results (Vernin et al. 2000) which is, at our best knowledge, the longest site testing campaign ever done measuring directly the $C_{\mathrm{N}}^{2}$ profiles (4 weeks equally distributed over the year). From the results of this campaign it was found that the average free atmosphere seeing in the austral summer is 0.49 arcsec and in the winter time is 0.87 arcsec. Moreover, the $\tau_{\mathrm{AO}}$ estimated in this campaign in the winter time is $7.25 \mathrm{msec}$ and in the summer time is $1.65 \mathrm{msec}$. The seasonal difference is so $5.6 \mathrm{msec}$ absolutely comparable with that found by our model in San Pedro Mártir.

\section{4. $\tau_{\mathrm{AO}}$ forecasting}

We proved that, neglecting the contribution of the $C_{\mathrm{N}}^{2}$ fluctuations, the $\tau_{\mathrm{AO}}$ difference between the winter and summer time is of about $5.48 \mathrm{msec}$. Probably, this difference is reinforced by the $5 / 3$ wind power (Eq. (2)). We find that, considering the contributions of the $C_{\mathrm{N}}^{2}$

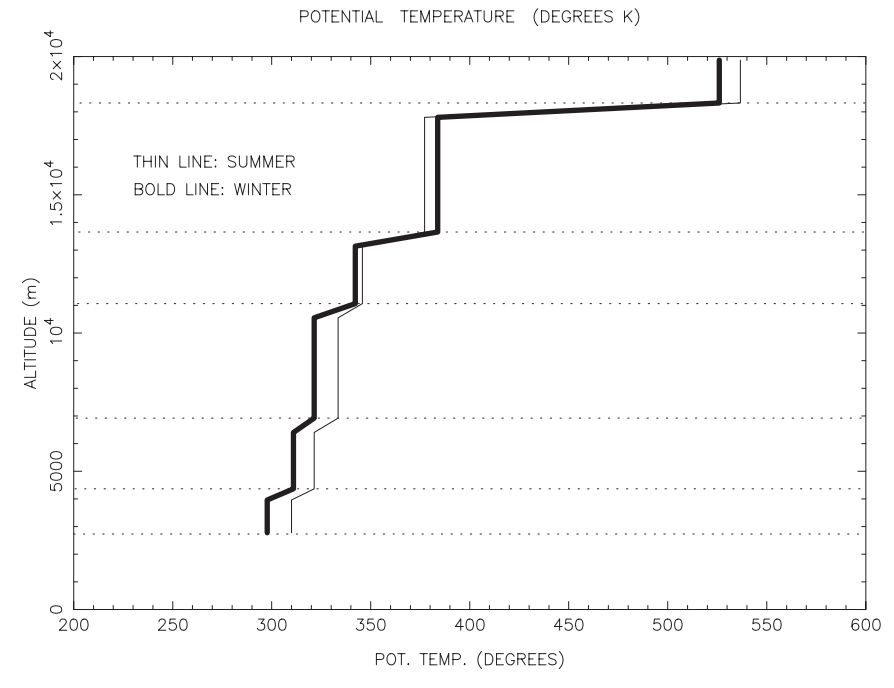

Fig. 4. Vertical potential temperature profiles of the ECMWF analysis averaged over the winter time (bold line) and over the summer time (thin line). The data are related to the $(31 \mathrm{~N}$, $116 \mathrm{~W})$ grid point

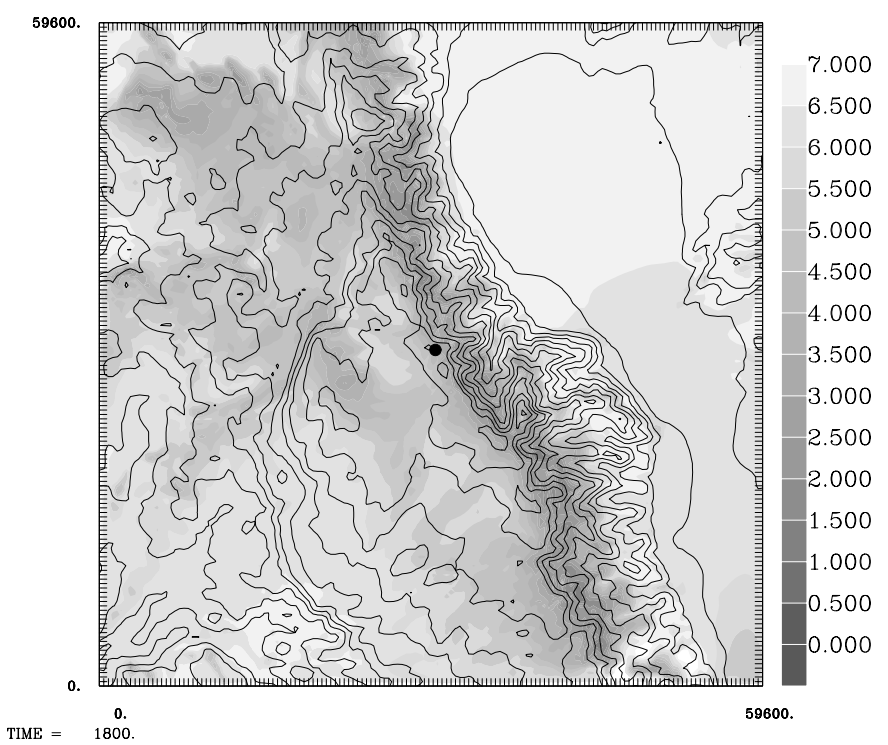

Fig. 5. $\tau_{\mathrm{AO}}$ map simulated above the San Pedro Martir Observatory (central black point) the 27 July 1997 night. The North is at the top of the figure. The black lines are the levels isolines 
fluctuations, this difference is statistically enhanced. At the same time, we saw in Sect. 3 that particular $C_{\mathrm{N}}^{2}$ and wind values can affect the $\tau_{\mathrm{AO}}$ and modify locally its seasonal characteristics. It is therefore very important, at the same time, to characterize the seasonal variability of $\tau_{\mathrm{AO}}$ and to forecast the $\tau_{\mathrm{AO}}$ values. The critical points related to the model ability to forecast $\tau_{\mathrm{AO}}$ in an operational mode are:

- the Meso-Nh reliability in simulating $C_{\mathrm{N}}^{2}$ profiles

- the representative level of the atmospheric flow of the initialization data.

We can characterize the $C_{\mathrm{N}}^{2}$ reliability comparing the dispersion of the measurements obtained with different instruments $\left(\Delta C_{\mathrm{N}}^{2}\right)$ with the dispersion between the average of measurements and simulations $\left(\Delta C_{\mathrm{N}}^{2 *}\right)$. In a previous article (Masciadri et al. 2000a) we proved that $\Delta C_{\mathrm{N}}^{2}$ and $\Delta C_{\mathrm{N}}^{2}{ }^{*}$ are comparable. Moreover, we recently tested a new method for the model calibration that seems to improve the Meso-Nh reliability. A preliminary result shows a difference between measurements and simulations of $8 \%$. A forthcoming article will describe these results in detail.

In order to characterize the initialization data quality we compared (Fig. 6) the analysis profiles of meteorological parameters $(p, T$ and $\boldsymbol{V})$ computed at 00:00 U.T. hours of the day $J$ with the forecasted profiles at 6 and 12 hours of the same parameters computed, respectively, at 18:00 and 12:00 U.T. hours of the day $(J-1)$. Both the analysis and the forecasts are computed in the AE grid point in an up-stream position respect to the San Pedro Mártir Observatory. We suppose that the analysis represent the "real" state of the atmospheric flow and we analyze the dispersion of the forecasts. Doing so we estimate how realistic are the initialization data. Table 4 shows, for each meteorological parameter, the relative error $(\bar{\varepsilon})$ between the analysis and the forecasts at 6 hours (first line) and the analysis and forecasts at 12 hours (second line). The third column is related to the high atmosphere [4-17.5] km and the fourth to the low atmosphere $[0-4] \mathrm{km}$. The results shown are computed considering the dispersion at each model level for 156 nights. The four analyzed parameters are used to initialize Meso-Nh. We can observe that the absolute temperature and the dew point temperature are well correlated. The wind intensity and direction show a larger dispersion. In general, we find a larger dispersion for the forecasts at 12 hours than for the forecasts at 6 hours. Above $4 \mathrm{~km}$ the largest wind intensity and direction average relative errors are, respectively, $14 \%$ and $10.36 \%$. In the low atmosphere the relative errors grow because the orographic effects are not well represented by the ECMWF climatological model. Anyway, the worst statistical dispersion is of $26.5(\%)$ which we judge acceptable for our present studies.

\section{Conclusions}

We used 156 wind intensity profiles provided by the ECMWF data bank evenly distributed in the year 1997

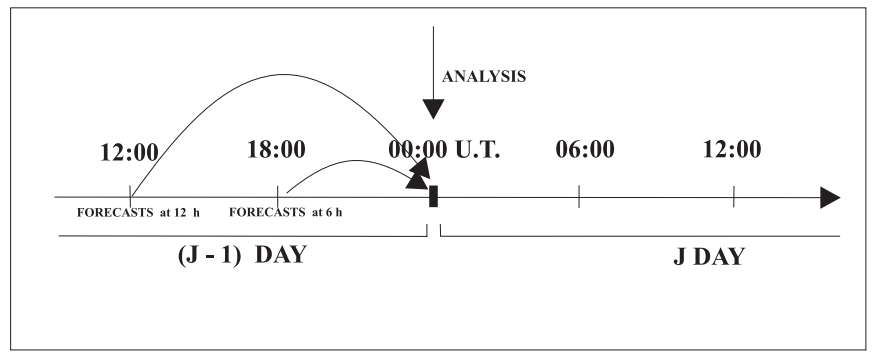

Fig. 6. Schematic drawing showing the meteorological initialization data (analysis and forecasts) used for the statistical analysis described in Sect. 4. The analysis at 00:00 U.T are compared to the forecasts at 6 hours (computed at 18:00 U.T. of the $(J-1)$ day) and forecasts at 12 hours (computed at 12:00 U.T. of the $(J-1)$ day)

and representative of the atmospheric flow over the San Pedro Mártir site to carry out a $\tau_{\mathrm{AO}}$ seasonal variability study. We proved that, using $C_{\mathrm{N}}^{2}$ profiles simulated by the non-hydrostatic model Meso-NH, and neglecting the high frequency fluctuations of the $C_{\mathrm{N}}^{2}$ profiles, we obtain typical $\tau_{\mathrm{AO}}$ values for the wavelength $\lambda=0.5 \mu \mathrm{m}$. Under this hypothesis, the $\tau_{\mathrm{AO}}$ amplitude seasonal variability at San Pedro Mártir site is of about $5.48 \mathrm{msec}$. We investigated the $C_{\mathrm{N}}^{2}$ seasonal variability (slow frequency variability) using $C_{\mathrm{N}}^{2}$ simulations provided by Meso-NH (4 nights in the summer time, 4 nights in the winter time). Our preliminary results show that such a variability, probably, exists and it has a tendency to enhance the previously reported $\tau_{\mathrm{AO}}$ difference. In Sect. 3 we have suggested a physical stochastic explanation for this phenomenon. Some $C_{\mathrm{N}}^{2}$ measurements done in the past (Vernin et al. 2000) confirm the $C_{\mathrm{N}}^{2}$ difference in winter and summer time at high altitudes. The interesting result of this paper is that the numerical models are indeed able to reproduce this effect.

We showed that, locally, the $C_{\mathrm{N}}^{2}$ can modify the $\tau_{\mathrm{AO}}$. This means that it is fundamental to be able to forecast $\tau_{\mathrm{AO}}$. We investigated so the Meso-Nh potential ability to do it. We analyzed the two more critical points: (1) the model reliability in simulating $C_{\mathrm{N}}^{2}$ profiles (2) that the initialization meteorological forecasting represent well the atmospheric flow over the site. Relating to the first point we can affirm that the average dispersion between the simulations and the average of the measurements is comparable to the dispersion between the measurements obtained with different instruments. Relating to the second point, we proved that the meteorological forecasts (at 6 and 12 hours) that we could use to initialize the model in an operational configuration, are well correlated to the analysis (considered here as the "real" atmospheric flow representation). The maximum average error (26.5\%) is obtained for the wind intensity between the forecasts at 12 hours and the analysis.

These results open new perspectives in the site testing studies and the flexible-scheduling of the telescope instruments and scientific programs. Ambitious projects 
Table 4. Estimation of the average relative errors computed between the analysis and the forecasts at 6 and 12 hours during the whole of 1997

\begin{tabular}{cccc}
\hline \hline Parameter & Forecasts & err (\%) $[4-17.5] \mathrm{km}$ & err (\%) $[0-4] \mathrm{km}$ \\
\hline Wind Intensity & 6 & 8 & 21.68 \\
& 12 & 14 & 26.5 \\
Wind Direction & 6 & 10.15 & 14.7 \\
& 12 & 10.36 & 15.70 \\
Absolute Temperature & 6 & 0.2 & 0.185 \\
& 12 & 0.3 & 0.265 \\
Dew point Temperature & 6 & 1 & 1 \\
& 12 & 1.18 & 1.2 \\
\hline \hline
\end{tabular}

to build a new class of telescopes now exist (OWL: Overwhelmingly Large Telescope - $D=100 \mathrm{~m}$, Hubin et al. 2000; CELT California Extremely Large Telescope - $D=30 \mathrm{~m})$. For these projects, excellent sites are necessary and numerical models could be used to choose the best of all possible sites. We found that, in the San Pedro Mártir site, the summer time is the potentially better period for $\tau_{\mathrm{AO}}$. This means that, the best scientific programs requiring high angular resolution could be scheduled in this period. Moreover, we remember that one of the critical points for the successful application of some of the adaptive optics techniques such as the tomographic one (Ragazzoni et al. 1999; Ragazzoni et al. 2000) or the Multiconjugated Adaptive Optics MCAO (Fusco et al. 1999; Fusco et al. 2000) is the characterization of the $C_{\mathrm{N}}^{2}$ during a long period and in a whole $3 \mathrm{D}$ domain around the telescope. Indeed, the optical turbulence has a typically non-uniform distribution in the atmosphere and the model indicates that different lines of sight can provide large differences in the seeing estimation (Masciadri et al. 2000b). The numerical modeling technique is, in our opinion, probably one of the best candidates for obtaining such a $3 \mathrm{D}$ characterization of the atmosphere turbulence. Some of the advantages with respect to other techniques are: it has no limitations in the vertical resolution, it does not suffer of exposure time limitations, it supplies $3 \mathrm{D} C_{\mathrm{N}}^{2}$ maps and $2 \mathrm{D}$ integrated parameters (as $\left.\tau_{\mathrm{AO}}\right)$ maps.

Acknowledgements. This work was support by the TIM (Telescope Infrarojo Mexicano) Project, the CONACYT grants (J32412E) and the DGAPA grants (IN118199). The Meso-Nh simulations were run on the Fujitsu VPP5000 supercomputer (CNRM - Meteo France - Toulouse, Fr). We thank P. Bougeault for kindly supplying CPU time for this study. We are grateful to P. Jabouille and J. Stein for helpful discussions about the Meso-Nh code. We are grateful to F. Angeles and L. Morales for providing the vegetation model implemented in the MesoNh model. Special thanks to A. Raga for useful discussions.

\section{References}

Alvarez, M. 1969, Reporte IA-UNAM

Avila, R., et al. 2000, Adaptive Optical System Technology, SPIE Proc., 4007, Munich 2000

Buscher, D. F. 1988, MNRAS, 235, 1203

Buscher, D. F. 1994, SPIE, 2200, 260

Davis, J., \& Tango, W. J. 1996, PASP, 108, 456

Echevarria, J., et al. 1998, RMxAA, 34, 47

Fusco, T., et al. 1999, Opt. Lett., 24, (N.21), 1

Fusco, T., et al. 2000, Adaptive Optical System Technology, SPIE Proc., 4007, Munich 2000

Greenwood, D. P. 1977, JOSA, 67, 390

Holton, 1992, An introduction to Dynamic Meteorology (Academic Press)

Hubin, N., et al. 2000, Adaptive Optical System Technology, SPIE Proc., 4007, Munich 2000

Lafore, J. P., et al. 1998, Ann. Geophys., 16, 90

Martin, F., et al. 2000, A\&A, in press

Masciadri, E., et al. 1999a, A\&AS, 137, 185

Masciadri, E., et al. 1999b, A\&AS, 137, 203

Masciadri, E., et al. 2000a, A\&A, in press

Masciadri, E., et al. 2000b, Interferometry in Optical Astronomy, SPIE Proc., 4006, Munich 2000

Nightingale, N. S., \& Buscher, D. F. 1991, MNRAS, 251, 155

Ragazzoni, R., et al. 1999, A\&AL, 342, L53

Ragazzoni, R., et al. 2000, Nature, 403, 54

Roddier, F., \& Lena, P. 1984, J. Opt. Paris, 15, 171

Roddier, F. 1981, Prog. Opt., XIX, 283

Roddier, F., et al. 1982, J. Opt. Paris, 13, 263

Tango, W. J., \& Twiss, R. Q. 1980, Prog. Opt., XVII

VanZandt, T. E., et al. 1978, Radio Sci., 13, 819

VanZandt, T. E., et al. 1981, 20th Radar Meteor. Conference - Boston, American Meteorological Society

Vernin, J., et al. 2000, Gemini Report, Web Page: http://www.gemini.edu/sciops/instruments /adaptiveOptics/ 